\title{
A Dual Centrifuge for Generating Low-Frequency Sinusoidal Accelerations
}

\author{
R. O. Smith, E. A. Willis, and J. S. Hilten
}

(July 10, 1962)

\begin{abstract}
This paper describes an experimental "dual centrifuge" in which an accelerometer being calibrated is carried around a circular path in a horizontal plane. If the instrument is constrained to have nonrotational motion, such as is provided by a parallel link device, sinusoidal excitation along its sensitive (horizontal) axis is obtained. The excitation obtainable is equivalent to linear excitation but at unusually low frequency and large displacement. For example, a machine has been built which has a frequency range from 0.5 to 30 cycles per second, a displacement (zero to peak) up to 12 inches, and develops an acceleration amplitude, useful for calibration, up to 100 gravity at 10 cycles per second and above.
\end{abstract}

\section{Introduction}

Because of the wide use of low-frequency accelerometers there is a need for a calibration system tailored to the requirements of these instruments. This necessity has led to the consideration of several methods of generating the required low-frequency accelerations. At low frequencies most systems suffer from one or more limitations, such as poor waveform, restricted amplitude, or excessive superimposed vibration.

The several systems for obtaining low-frequency sinusoidal calibration have been reviewed, which may be classified as (1) electrodynamic, (2) transient, or (3) mechanical shakers.

The electrodynamic shaker is undoubtedly the most useful calibrator for laboratory use. However, it usually suffers from insufficient amplitude at low frequencies. A typical example of a low-frequency electrodynamic shaker has a frequency range of 0.35 to $500 \mathrm{c} / \mathrm{s}$ but provides a maximum displacement of only 2 in. (double amplitude), whereas about 20 in. displacement is required to develop $\mathrm{l} g$ at $1 \mathrm{c} / \mathrm{s}$.

Transient shakers usually take the form of a cantilever spring upon whose free end the instrument under test is fixed. They suffer from the fact that the excitation is transient, so that it is difficult to correlate excitation amplitude with response. In addition each shaker is usually restricted to a single frequency and several shakers are required to cover any appreciable range.

The mechanical shakers reviewed may be subdivided into three classifications: (1) four-bar linkages, (2) inertial shakers, and (3) rotary calibrators. Four-bar linkages, e.g., scotch vokes and slidercrank mechanisms, cannot generate a truly sinusoidal test motion because of the varying effective moment of inertia. In order to get the requisite stiffness in the reciprocating parts it is required that a relatively large mass be subjected to the test motion so that the sinusoidally varying inertial forces are necessarily large. If the test motion is to be nearly sinusoidal it is required that the angular velocities of the rotating parts be nearly uniform and it will therefore be required that the rotating members have a high moment of inertia, and a bulky, massive device results.

An inertial shaker suitable for calibration purposes has been designed by the Engineering Mechanics Section of the Bureau [1] ${ }^{1}$ which consists of a springmass driven by an eccentric weight. Its frequency range is 20 to $110 \mathrm{c} / \mathrm{s}$. Inertial shakers of lower frequency are rarely used for calibration because of poor waveform.

A rotary dynamic calibrator which uses the earth's field for excitation has been devised by Wildhack and Smith [2]. This device is limited in acceleration amplitude to a maximum of $1 \mathrm{~g}$ at all frequencies. It is presently in use by the Mechanical Instruments Section of the Bureau over the frequency range 1 to $30 \mathrm{c} / \mathrm{s}$.

The dual centrifuge, which uses its own centrifugal field for excitation, was described by Woolard in 1939 [3]. It consists essentially of a small turntable mounted on a large one, each turning in a horizontal plane. The instrument under test is mounted on the small table. When the turntables have constant angular velocities the seismic mass of the accelerometer responds to a sinusoidally varying component of the centrifugal force field generated by the rotation of the large table. Woolard published an equation showing the acceleration amplitude in terms of angular velocities, original displacement, and relative displacement of the seismic mass, but did not publish a complete mathematical analysis of the response. However, he did point out that when the small table turns backwards so that it has zero absolute rotation, the accelerometer is submitted to sinusoidal acceleration which may be of large amplitude. This is the practically important feature of the dual centrifuge and one that has been generally overlooked.

The following discussion, which describes a mechanical configuration similar to Woolard's design, and develops the equation of motion of the seismic mass, shows the dual centrifuge to be a useful tool for dynamic calibration, particularly when a large amplitude is required at low frequencies.

\footnotetext{
1 Figures in brackets indicate the literature references at the end of this paper.
} 
2. Analysis of the Response of a Linear 1-Degree of Freedom Seismic Accelerometer to General Plane Motion Excitation

Although the conclusion of this paper is that the useful form of the dual centrifuge is that one in which the small table has zero absolute rotation, a general analysis of the motion without this limitation is instructive.

In the general case, the housing of the instrument under test will have motion of 3 degrees of freedom, i.e., two of translation and one of rotation. The physical situation is shown in figure 1. The large turntable turns with an absolute angular velocity $\omega_{0}$ about its center 0 . The small table rotates about point $p$ with constant absolute angular velocity $\omega_{p}$, and an angular velocity $\Omega=\omega_{p}-\omega_{0}$ relative to the large table. The point 0 is chosen as the origin of a plane nonrotating coordinate system, with positive directions chosen according to usual convention. Let the radius from 0 to $p$ be $R_{0}$. In general, the test position $p^{\prime}$ of the seismic mass $m$ will not coincide with $p$. If $a-b$ represents the direction of the sensitive axis, then $p^{\prime}$ is located an amount $d$ from $p$, perpendicular to $a-b$, and an amount $e$ parallel to $a-b$. Response of the instrument consists of displacement $S$ along $a-b$ and the equation of motion is therefore in terms of $S$.

The following assumptions apply to the analysis:

(1) Plane horizontal motion,

(2) Point 0 is fixed,

(3) $R_{0}, \omega_{0}$, and $\omega_{p}$ are constant,

(4) The instrument has no transverse response,

(5) The instrument is a linear 1-degree of freedom system,

(6) Accelerometer damping is viscous.

Referring to figure 1 , it will be seen that if the seismic mass $m$ is constrained to motion along the axis $a-b$ the motion of the mass may be described by the following coordinate equations in which $s$,

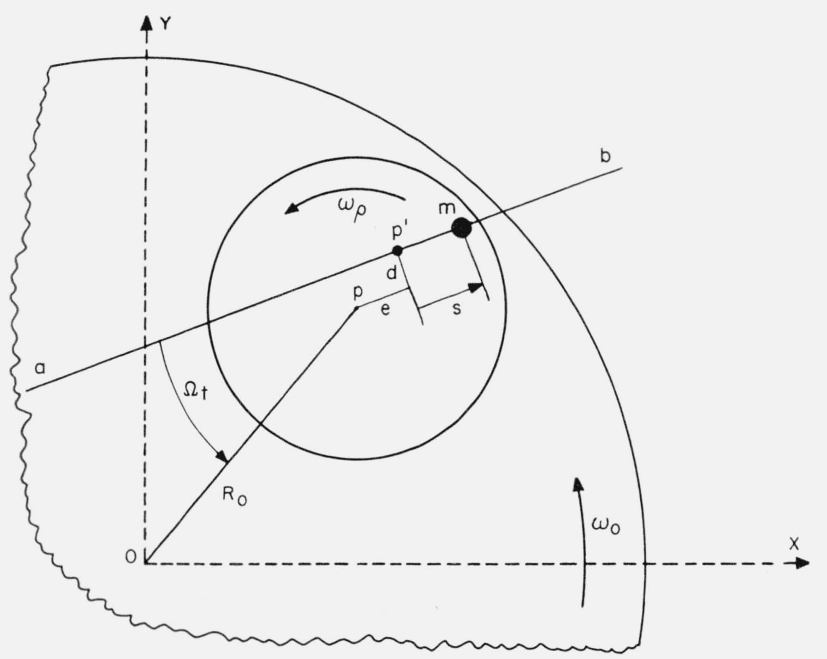

Figure 1. Schematic diagram of the dual centrifuge. $s$, and $\ddot{s}$ are relative to the instrument case:

$$
\begin{aligned}
& x=R_{0} \cos \omega_{0} t-d \sin \omega_{p} t+(e+s) \cos \omega_{p} t \\
& y=R_{0} \sin \omega_{0} t+d \cos \omega_{p} t+(e+s) \sin \omega_{p} t .
\end{aligned}
$$

Double differentiation shows that the coordinate equations which describe the acceleration of $m$ are:

$$
\begin{aligned}
\ddot{x}= & -R_{0} \omega_{0}^{2} \cos \omega_{0} t+d \omega_{p}^{2} \sin \omega_{p} t-(e+s) \omega_{p}^{2} \cos \omega_{p} t \\
& -2 \dot{s} \omega_{p} \sin \omega_{p} t+\ddot{s} \cos \omega_{p} t \\
\ddot{y}= & -R_{0} \omega_{0}^{2} \sin \omega_{0} t-d \omega_{p}^{2} \cos \omega_{p} t-(e+s) \omega_{p}^{2} \sin \omega_{p} t \\
& +2 \dot{s} \omega_{p} \cos \omega_{p} t+\ddot{s} \sin \omega_{p} t .
\end{aligned}
$$

The acceleration along the axis $a-b$ to which the mass $m$ is subjected is the sum of the projections of these coordinate components along $a-b$, i.e., acceleration $=\ddot{x} \cos \omega_{p} t+\ddot{y} \sin \omega_{p} t$ or acceleration $=\ddot{s}-(e+s) \omega_{p}^{2}-R_{0} \omega_{0}^{2} \cos \Omega t$.

So that the equation of motion may be derived:

$$
m \ddot{s}+c \dot{s}+k s=m R_{0} \omega_{0}^{2} \cos \Omega t+m(e+s) \omega_{p}^{2} .
$$

If $\omega_{n}=\sqrt{\frac{k}{m}}$ and $p=c / c$ critical when $c$ critical $=2 \sqrt{m k}$, this equation can be reduced to :

$$
\ddot{s}+2 p \omega_{n} \dot{s}+\left(\omega_{n}^{2}-\omega_{p}^{2}\right) s=R_{0} \omega_{0}^{2} \cos \Omega t+e \omega_{p}^{2} .
$$

The solution of eq $(6)$ is:

$$
\begin{aligned}
s= & A e^{-\omega_{n} t\left(p+\sqrt{p^{2}-1+\frac{\omega_{p}^{2}}{\omega_{n}^{2}}}\right)}+B e^{-\omega_{n} t\left(p-\sqrt{\left.p^{2}-1+\frac{\omega_{p}^{2}}{\omega_{n}^{2}}\right)}\right.} \\
& +\frac{R_{0} \omega_{0}^{2}\left[\left(\omega_{n}^{2}-\Omega^{2}-\omega_{p}^{2}\right) \cos \Omega t+2 p \omega_{n} \Omega \sin \Omega t\right]}{\left(\omega_{n}^{2}-\omega p^{2}-\Omega^{2}\right)^{2}+4 p^{2} \omega_{n}^{2} \Omega^{2}}+\frac{e \omega_{p}^{2}}{\omega_{n}^{2}-\omega_{p}^{2}} .
\end{aligned}
$$

The steady state portion of eq $(7)$ reduces to:

$$
s=\frac{R_{0} \omega_{0}^{2} \cos (\Omega t-\phi)}{\left[\left(\omega_{n}^{2}-\omega_{p}^{2}-\Omega^{2}\right)^{2}+4 p^{2} \omega_{n}^{2} \Omega^{2}\right]^{1 / 2}}+\frac{e \omega_{p}^{2}}{\omega_{n}^{2}-\omega_{p}^{2}}
$$

in which

$$
\tan \phi=\frac{2 p \omega_{n} \Omega}{\omega_{n}^{2}-\omega_{p}^{2}-\Omega^{2}}
$$

It should be noted that in the form of the dual centrifuge in which $\omega_{p}=0$, i.e., $\Omega=-\omega_{0}$, eq (7) takes a form almost identical with the familiar equation for a spring-mass accelerometer subject to sinusoidal linear motion

$$
\begin{aligned}
& s=A e^{-\omega_{n} t\left(p+\sqrt{p^{2}-1}\right)}+B e^{-\omega_{n} t}\left(p-\sqrt{p^{2}-1}\right) \\
& +\frac{R_{0} \omega_{0}^{2}\left[\left(\omega_{n}^{2}-\Omega^{2}\right) \cos \Omega t+2 p \omega_{n} \Omega \sin \Omega t\right]}{\left(\omega_{n}^{2}-\Omega^{2}\right)^{2}+4 p^{2} \omega_{n}^{2} \Omega^{2}}
\end{aligned}
$$

In which $\Omega \equiv-\omega_{0}$. 
The steady state portion may be written:-

$$
s=\frac{-R_{0} \omega_{0}^{2} \cos \left(\omega_{0} t-\phi\right)}{\sqrt{\left(\omega_{n}^{2}-\omega_{0}^{2}\right)^{2}+4 p^{2} \omega_{n}^{2} \omega_{0}^{2}}}
$$

From the foregoing it is evident that for the special case in which the instrument has only translational motion, the motion of $m$ is simple sinusoidal and hence may be directly compared with data using an electrodynamic shaker. In fact, for a given instrument, data from the double centrifuge and the electrodynamic shaker may be used together to construct a single response curve (see figs. 7, 8, and 9).

\section{Limitations of the Dual Centrifuge}

Consideration of the response of a linear, single degree of freedom system to the general case of the dual centrifuge in which the angular velocity of the little table is independent of that of the large table indicates that this form of the equipment is not attractive for accurate calibration. Examination of the response equation (7) shows that the magnitude of the transient at any instant depends upon $\omega_{p}$ as well as upon the instrument natural frequency $\omega_{n}$ and the instrument damping $p$. When $p^{2}+\frac{\omega p^{2}}{\omega_{n}^{2}}<1$, the transient is oscillatory. At $p^{2}+\frac{\omega p^{2}}{\omega_{n}^{2}}=1$, the transient is critically damped and when $p^{2}+\frac{\omega p^{2}}{\omega_{n}^{2}}>1$, it is over-damped. This latter suggests that at very large values of the ratio $\frac{\omega_{p}}{\omega_{n}}$ the decay of the transient may be so slow as to prevent the practical attainment of the steady state condition. Moreover, examination of the steady bias portion of eq (8) shows that, even after steady state conditions have been attained, there is a range of $\omega_{p}$ over which the seismic mass will be frozen against the instrument stops, the frequency range over which this condition will exist depending on the magnitude of the offset $e$ in figure 1 .

Without further evaluation of either of the above conditions, a decisive consideration is found by examination of the periodic portion of eq (8) which discloses that the condition of resonance will be realized whenever $\omega_{p}+\Omega=\omega_{n}$, so that a frequencyresponse curve of the instrument can be drawn only if the condition $\omega_{p}=$ constant, i.e., $\omega_{0}+\Omega=$ constant, can be maintained. Since the frequency-response curve is the objective of sinusoidal input testing, this limitation appears to make use of the general case of the dual centrifuge unprofitable. Accordingly the test machine which will be described in the next section was made to operate with $\omega_{p}=0$ so that eq (10) is applicable.

It is to be noted that, since a transverse axis undergoes the same motion as the sensitive axis, the instrument under test is subjected to a sinosoidal transverse excitation of the same amplitude and frequency as the excitation along the sensitive axis. If the transverse response of the instrument is linear and independent of the principal (axial) response, the transverse response will be sinusoidal and will lag, or lead, the axial response by $90^{\circ}$. Under this circumstance the error in calibration can easily be estimated by assuming the electrical output of the instrument to be proportional to the vector sum of the axial and transverse responses. At a transverse response of 5 percent of the axial response, the error is thus found to be about 0.12 percent. In some instruments, however, the response along one transverse axis is dependent on the axial response, increasing with the axial response. For this case the error is not easily computed as the phase between the axial and transverse responses will depend upon this rate of increase of transverse response. Moreover, in this case, the transverse response is most likely to be nonsinusoidal. However, it appears that instruments with an appreciable dependence of transverse on axial response along more than one transverse axis are rare.

\section{Description of the Dual Centrifuge}

In view of the obvious advantage in having available for instrument test purposes the large acceleration amplitude at low frequency that can be generated with the dual centrifuge and in view of the great simplicity of the dual centrifuge when it operates with $\omega_{p}=0$, a dual centrifuge of this type has been built.

A pilot model was first designed, constructed, and tested in order to investigate the potential performance of this type of apparatus. This was subsequently replaced with an improved version suitable for continuous laboratory use. The pilot model dual centrifuge shown in figures 2 and 3 consists essentially of a small turntable (2) mounted on a large turntable (1) by means of a roller bearing cartridge (6) and a radius arm (4). The instrument (3) is mounted on the small turntable (2). Its leads are brought to the overhead arm (15), no slip rings being required. Since the pulleys which drive the small table are all the same size, the absolute angular velocity of the small turntable is controlled only by the coaxial control pulley (14). In this case the control pulley was fixed and hence the small turntable has zero absolute angular velocity. Motion is transmitted through a system of open timing belts and pulleys $(7,8)$. The main table is turned by a motor (11) which is a shunt wound d-c motor with armature rheostat speed control. Power is transmitted via belt drive (12). The roller bearing cartridge of the countershaft (5) serves to center radius arm (4). The radius arm assembly is made symmetrical by the addition of a small balance weight (9). Thus the effective radius from the center of the large turntable to the center of the small turntable may be varied without altering the dynamic balance of the machine. Counterweight (10) serves to balance the combined effect of the radius arm assembly and the countershaft assembly. In 


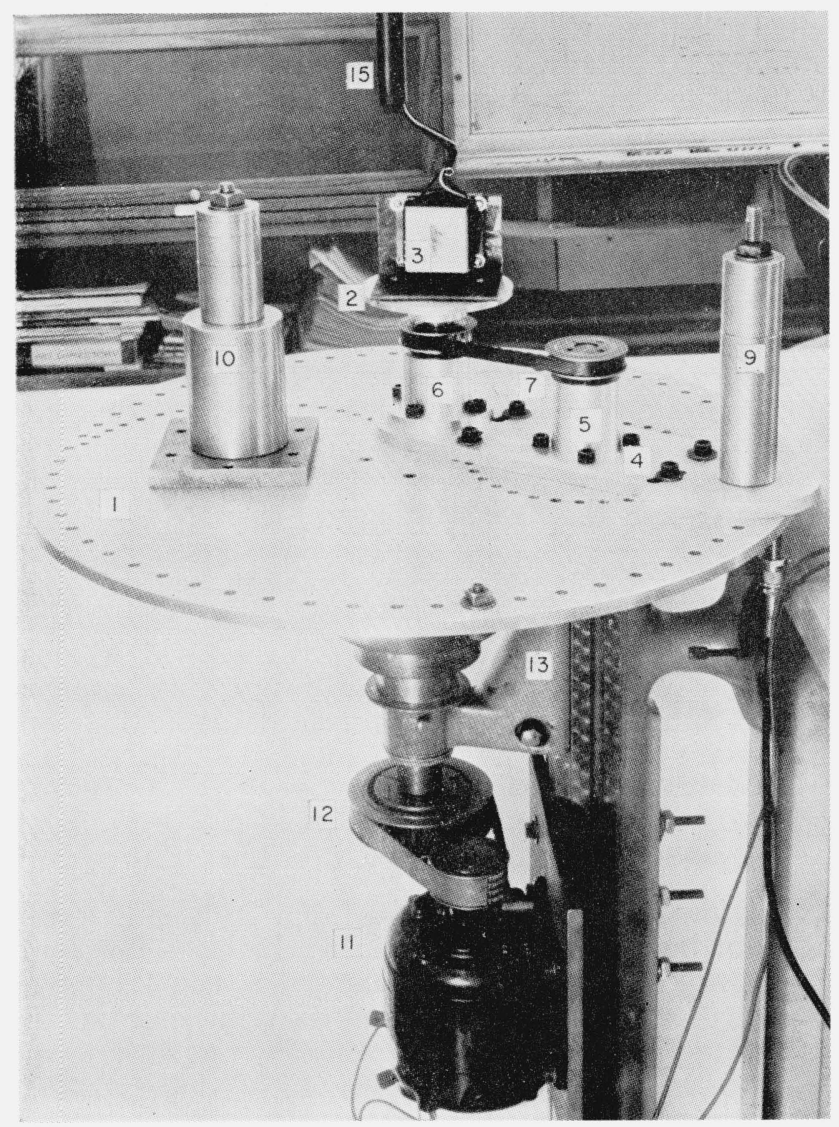

Figure 2. Experimental dual centrifuge.

preparation for a calibration, the accelerometer is balanced by adding an equal weight to the small counterbalance (9) and twice that to the large counterbalance (10). The whole assembly is supported by the machine frame (13).

The machine shown in figure 4 conforms with the basic design illustrated in figures 2 and 3 , but has an extended amplitude and frequency range. It has a frequency range from 0.5 to $30 \mathrm{c} / \mathrm{s}$, a displacement amplitude range up to 12 in., and an acceleration amplitude range up to $100 \mathrm{~g}$ at $10 \mathrm{c} / \mathrm{s}$ and higher suitable for calibration purposes. The zero to peak amplitude of the displacement is measured to within \pm 0.02 in. by means of two measurements of the distance from a fiducial line on the frame to the edge of the small table. The angular velocity $\omega_{0} \equiv-\Omega$ is measured by counting on an electronic events per unit time meter the number of pulses from a small generator driven by the main shaft which develops 600 pulses for each revolution. If the count is over a period of 10 sec the accuracy with which the average velocity can be determined is \pm 1 part in 6,000 at 1 rps and with better accuracies at higher velocities. Errors in velocity measurement due to a progressive change in velocity can be minimized by observing the velocity over a period of time both before and after as well as during the calibration. Errors in calibration due to wow and to flutter at any frequency other than the frequency of test can be

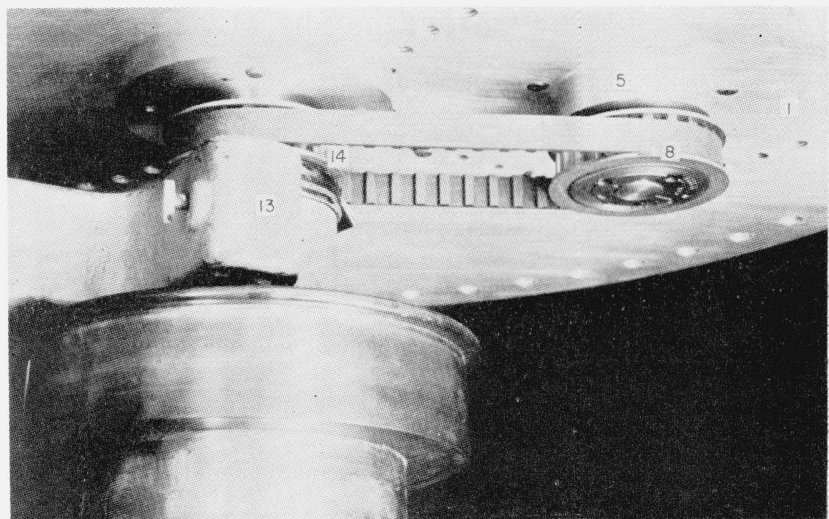

Figure 3. Experimental dual centrifuge.

View of lower control pulleys

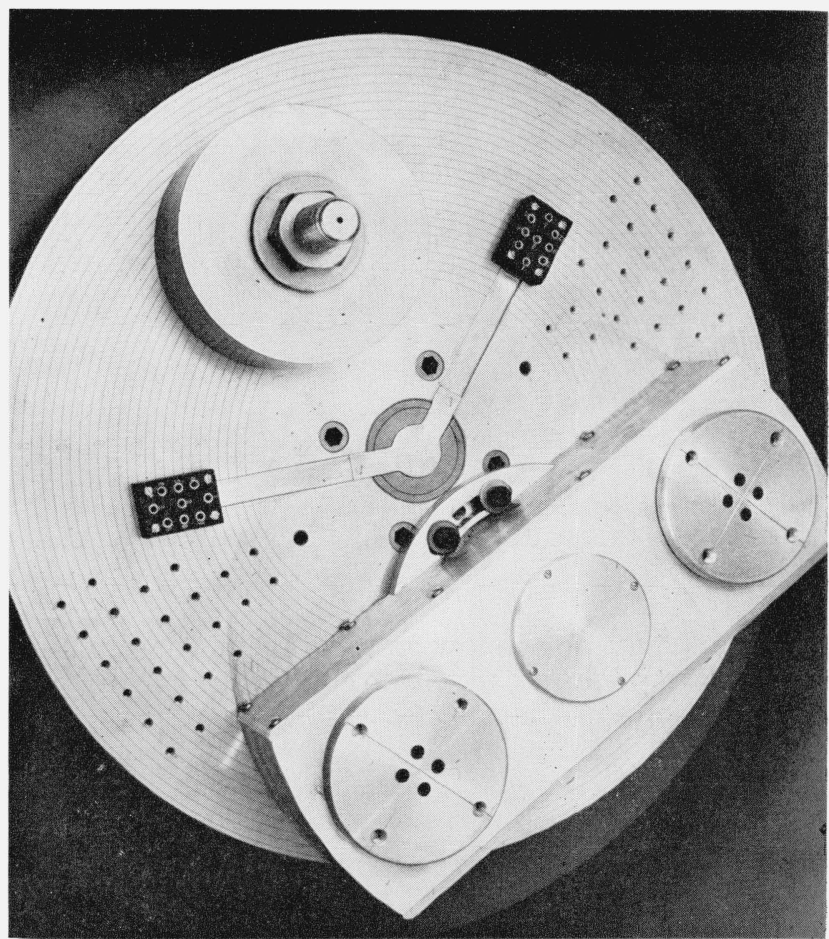

Figure 4. Dual centrifuge.

detected as a deviation from sinusoidal wave shape of the response of the instrument under test. It is estimated that in practice the total error in velocity measurement does not exceed \pm 0.02 percent of the velocity at any velocity above 1 rps.

An additional source of error is found in the fact that even the best rotating devices are not in perfect dynamic balance. The machine pictured in figure 4 has a critical speed around 22 rps, varying with the load, near which no calibrations have been made. There is some unwanted sinusoidal vibration due to dynamic unbalance at any frequency of test, but an independent measurement of it can be made with a piezo-electric accelerometer, which has no zero 


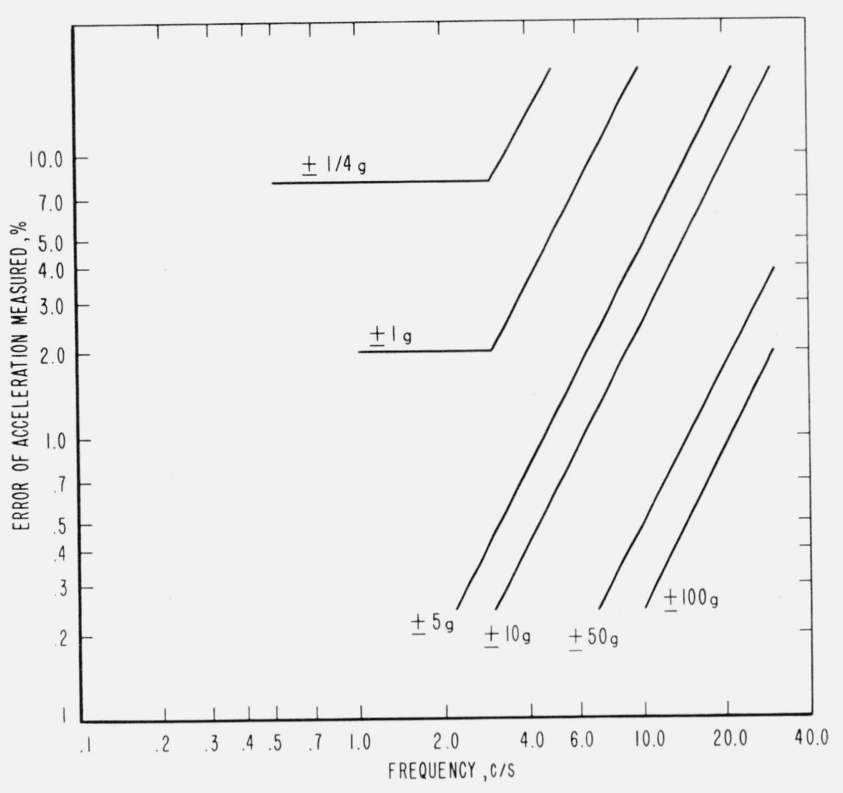

Figure 5. Dual centrifuge calibration accuracy of sinusoidal linear accelerations.

frequency response, mounted to the big table with its axis along a radius. The response of this instrument is then a measure of the unwanted vibration. Its phase relation to the response of the instrument under test is unknown, so that it can only be said that the sum of the error due to this vibration plus any other periodic errors due to either vibration, flutter or wow, or similar source does not exceed the response of the monitoring piezo-electric instrument to within the accuracy of the monitoring instrument. In the case of the machine pictured in figure 4 no such error greater than $\pm 0.02 g$ has been observed during any test.

The estimated calibration accuracy attained with this device over the acceleration range $\pm 1 / 4$ to $\pm 100 \mathrm{~g}$ and the frequency range 0.5 to $30 \mathrm{c} / \mathrm{s}$ is shown graphically on figure 5.

The phase of the response can be measured to within an estimated $\pm 5^{\circ}$ by recording the output of a photocell excited by a fixed light and a mirror attached to the large table on a dual trace oscillograph with the response of the instrument under test.

\section{Experimental Results}

Figures $6,7,8$, and 9 show the results of calibrations on the dual centrifuge. They appear to be typical frequency-response curves. Figure 6 shows

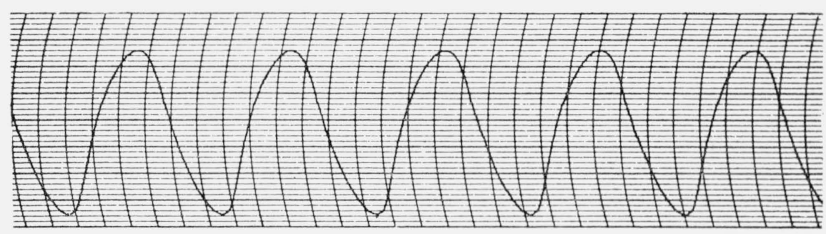

FiguRE 6. Output of potentiometer-type accelerometer under dual centrifuge excitation at $0.86 \mathrm{c} / \mathrm{s}$ and $0.5 \mathrm{~g}$ (zero peak).

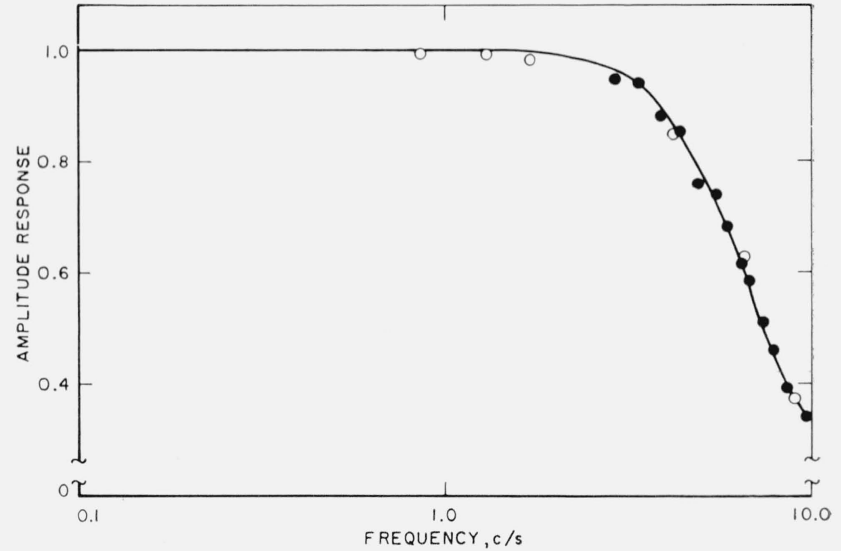

FiguRE 7. Frequency response of potentiometer-type accelerometer.

Theoretical curve for a linear system of one degree of freedom with a damping ratio of 0.75 and a natural frequency of $6.0 \mathrm{c} / \mathrm{s}$.

, excitation by electromagnetic shaker

0 , excitation by dual centrifuge
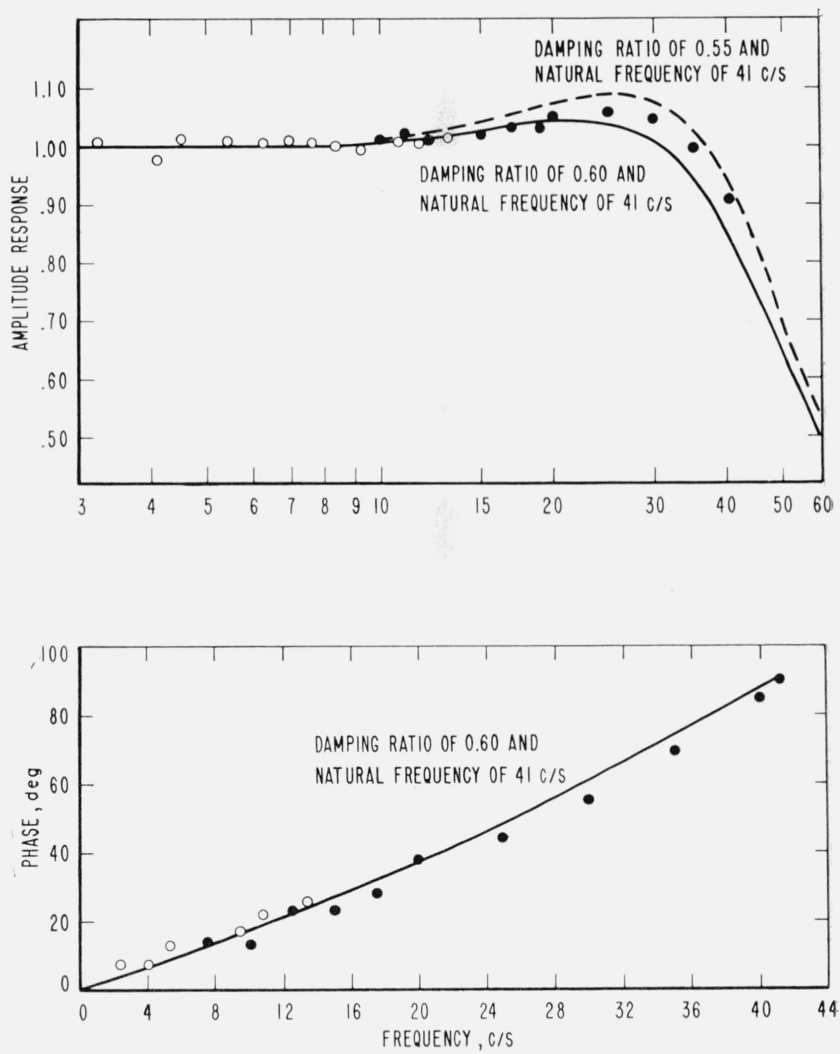

Figure 8. Frequency response of bonded strain-gage type accelerometer.

, excitation by electromagnetic shaker $\bigcirc$, excitation by dual centrifuge

the response of a potentiometer type acceleration transducer when tested on the experimental equipment pictured in figures 2 and 3 , as recorded by a pen writing oscillograph. Figure 7 shows the amplitude 


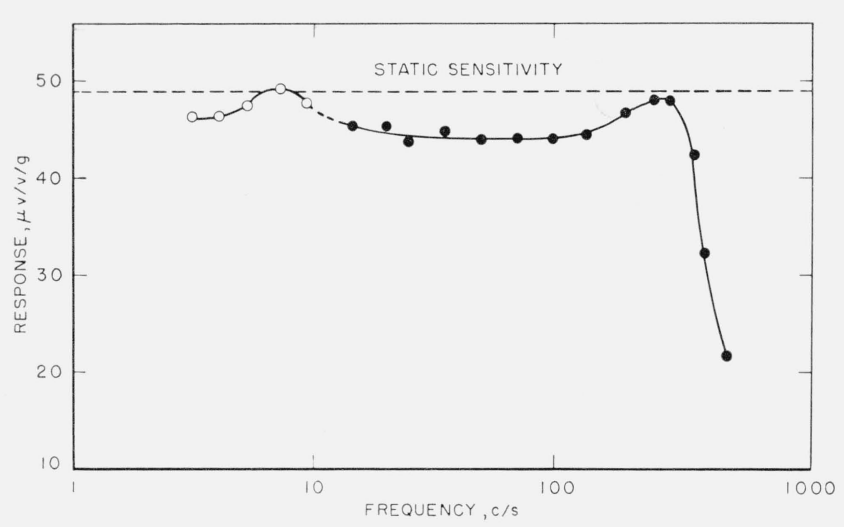

Figure 9. Frequency response of unbonded strain-gage type accelerometer.

, excitation by electromagnetic shaker $\bigcirc$, excitation by dual centrifuge response of the same instrument obtained by calibrations on the experimental equipment compared with the results of calibrations on an electromagnetic shaker. Figure 8 shows the frequency response, both amplitude and phase, of a bonded strain gage accelerometer determined by calibrations on the equipment pictured in figure 4 and by calibrations on an electromagnetic shaker.

Figure 9 shows the amplitude response of an unbonded strain gage accelerometer from calibrations on the equipment pictured on figure 2 and on an electromagnetic shaker.

\section{References}

[1] S. Levy, A. E. McPherson, and E. V. Hobbs, J. Research NBS 41, 359 (1948)

[2] W. A. Wildhack and R. O. Smith, ISA Paper No. 54-40-3.

[3] A. W. Woolard, Instruments 12, 322 (Dec. 1939).

(Paper 66C4-111) 